\title{
Congenital irreducible atlantoaxial dislocation associated with cervical intramedullary astrocytoma causing progressive spastic quadriparesis
}

\author{
Anooj Chatley, Awadesh K. Jaiswal, Manoj Jain ${ }^{1}$, Sanjay Behari \\ Departments of Neurosurgery and ${ }^{1}$ Pathology, Sanjay Gandhi Postgraduate Institute of Medical Sciences, Lucknow, India
}

\begin{abstract}
Simultaneous presence of congenital irreducible atlantoaxial dislocation (AAD) and cervical intramedullary astrocytoma has not been previously described and may cause disabling myelopathy. This 55-year-old lady presented with suboccipital pain, spastic quadriparesis, Lhermitte's phenomenon and sphincteric disturbances. Lateral radiographs and magnetic resonance imaging showed irreducible $A A D$, occipitalized atlas, C2-3 fusion, and,an intramedullary tumor from C2-5 level iso-to-hypointense, non-enhancing, except in a small segment in the dorsal $\mathrm{C} 2$ level.
\end{abstract}

A suboccipital craniectomy with C2-5 laminectomy revealed a greyish-white tenacious tumor. The tumor was decompressed using a C2-5 midline myelotomy and duroplasty. An occipitocervical lateral mass fixation was performed. Histopathology revealed a low-grade astrocytoma. At three-month follow-up, her spasticity had decreased and quadriparesis and sphincteric disturbances were persisting. Postoperative lateral radiographs and intrathecal contrast CT scan showed a stable occipitocervical construct. Thus, the suboccipital craniectomy and laminectomy with midline myelotomy and duroplasty facilitated space for progressively expanding intramedullary astrocytoma with irreducible $A A D$; the lateral mass fixation provided stability at the craniovertebral junction.

Key words: Atlantoaxial dislocation, intramedullary tumor, spinal cord astrocytoma.

\section{Introduction}

We report a rare case having congenital irreducible atlantoaxial dislocation (AAD), occipitalized atlas and C2-3 fusion associated with a cervical intramedullary low-grade astrocytoma leading to progressive spastic quadriparesis with sphincteric dysfunction. The radiological features and surgical strategies for simultaneously dealing with the two entities are described.

\section{Case Report}

A 55-year-old lady presented with suboccipital pain, progressive spastic quadriparesis and Lhermitte's phenomenon with hesitancy and precipitancy of micturition for one year. On examination, she had wasting of left forearm flexor, thenar and hypothenar muscles, spastic quadriparesis Grade IV and absence of left triceps and supinator jerks with hyperreflexia of all other deep tendon jerks. Her plain lateral radiographs of the craniovertebral junction (CVJ) in flexion and extension [Figure 1A and B] and T1-weighted magnetic resonance (MR) image revealed an AAD with occipitalized atlas, C2-3 fusion, asymmetrical occipitoC1-C2 lateral joints with a C2 to C5 level iso- to mildly hypointense tumor [Figure 2A]. On T2-weighted images, foramen magnum narrowing was associated with T2 intramedullary signal changes at C1-2 level. The cord was expanded by a dorsal, hyperintense lesion that extended from the mid C2 vertebral body level to C5-6 disc level [Figure 2B] with a small area of contrast enhancement at C2 level [Figure 2C]. The MR intensity pattern suggested a C2-C5 spinal cord astrocytoma.

At surgery, in prone position, a suboccipital craniectomy and a C2-5 laminectomy were performed. The expanded spinal cord had a grayish, tenacious avascular appearance. After C2-5 midline myelotomy, tumor decompression was done using ultrasonic suction aspirator. A duraplasty was performed using a fascia lata graft. Then, bilateral occipito-cervical Vertex plates and 


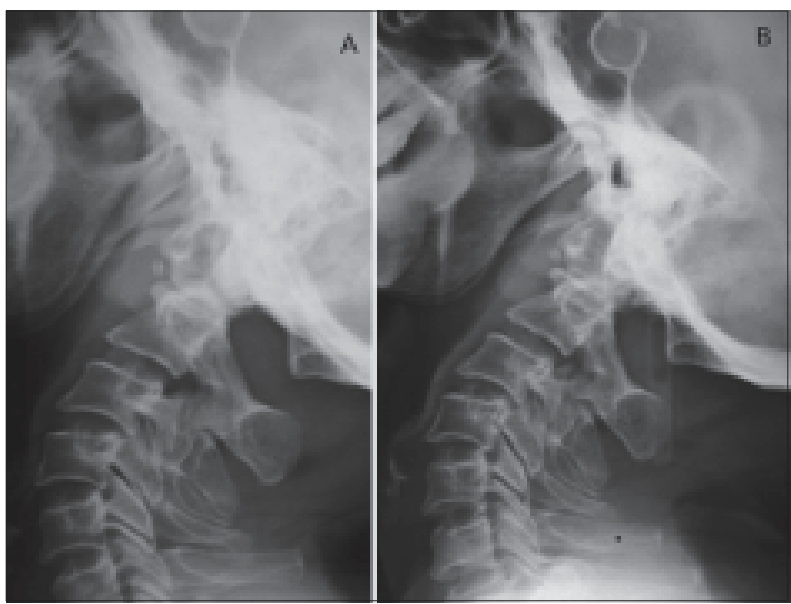

Figure 1: Lateral radiograph of the craniovertebral junction in (A) Flexion, and (B) Extension showing an irreducible atlantoaxial dislocation with occipitalized atlas

screws (Meditronics) contoured to the occipitocervical curvature with onlay bone grafts were fixed under fluoroscopic guidance to the occiput and C1-2 lateral masses. The patient was kept on overnight elective ventilation. The histopathology revealed a low-grade astrocytoma having round to oval hyperchromatic nuclei on a fibrillary background and absence of mitotic activity [Figure 3]. At follow-up after three months, her spasticity had decreased, her quadriparesis and left upper limb wasting and sphincteric disturbances were persisting. Her postoperative lateral radiograph of the cervical spine [Figure 4a] and sagittal reconstructed images of intrathecal contrast computed tomographic scan [Figure 4b] revealed the decompressed posterior margin of the foramen magnum and C2-5 laminectomy with a stable occipitocervical construct.

\section{Discussion}

\section{Pathogenesis and radiological features}

The congenital irreducible AAD in our patient was associated with other occipital and upper cervical sclerotomal defects like assimilated atlas, C2-3 fusion and asymmetrical occipitocervical facet joints. ${ }^{[1,2]}$ The recurrent flexion-extension of the neck causing tenting of the upper cervical cord against the posteriorly directed odontoid; the assimilated and stenosed atlantal canal diameter leading to foramen magnum compromise; and, the diffusely infiltrating low-grade astrocytoma expanding the cord from the C2-C5 level were all responsible for the progressive myelopathy that our patient developed. ${ }^{[1-4]}$

An intramedullary ependymal cyst at the C2-3 level with associated irreducible AAD causing spastic quadriparesis has been previously reported. The cyst was well-circumscribed with characteristic cerebrospinal fluid (CSF) density and non-enhancement on contrast. ${ }^{[5]}$ Goel et al., have reported a "waist-like"

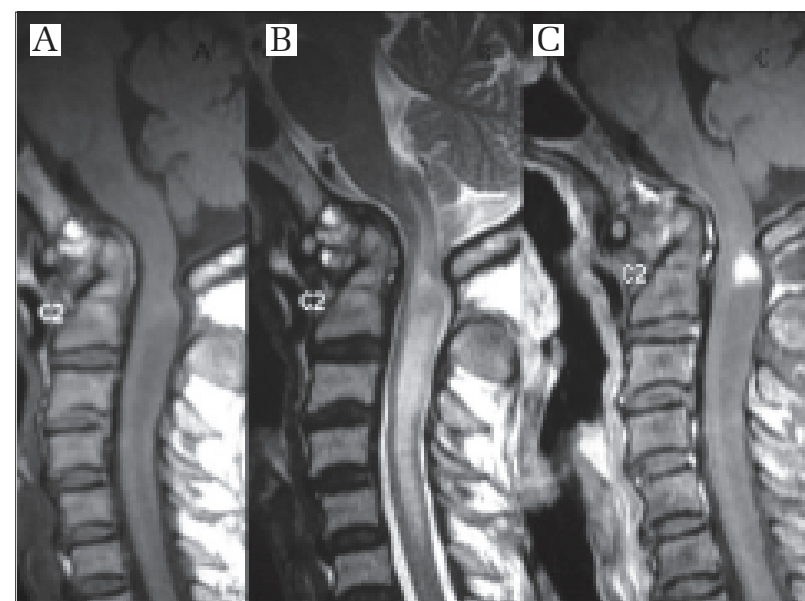

Figure 2: (A) T1 sagittal MR image: AAD with tenting of the cervicomedullary junction against odontoid. There is expansion of the cervical cord from the C2 to C5 level. T2-weighted sagittal MR image (B) showing at the foramen magnum level, T2 intramedullary signal changes within cord with anterior and posterior bony compression. At subaxial level, the cord is expanded by a hyperintense lesion. Contrast enhanced sagittal image (C) reveals a small area of contrast enhancement at C2 level. Rest of the expanded cord segments appeared iso to mildly hypointense without contrast enhancement

syringomyelia (with CSF-like intensity) in three infants having mobile and congenital AAD. They cautioned that in atleast two of these cases, the syringomyelia was earlier diagnosed to be an intramedullary tumor. ${ }^{[6]}$ The syrinx was present in the center of the cord with well-defined margins, mainly at the C1-2 level; and, at the site of maximum bony compression showing the "waist". ${ }^{[6]}$ In our patient, however, the C2-5 astrocytoma was below the level of the coexisting AAD. It was nearly isointense to the cord on T1-weighted images, more dorsally placed, and without a clear demarcation from the normal cord. The T2-weighted image showed its infiltrative nature; and, at the upper end of the lesion, there was a characteristic contrast enhancement. The diffusion weighted images showed a hyperintense signal indicating a solid tumor rather than a syrinx. The small nodular enhancement seen in our patient may also be seen in patients harboring spinal cord hemangioblastomas or granulomas. ${ }^{[3,4]}$ In the latter cases, cord expands both below and/or above the lesion; and, the intensity of the cord at the level of the widened segments does not differ from that of the normal cord. In our patient, however, the T2-weighted images showed the focal cord expansion to be distinct from the normal cord. In the present study, majority of the intramedullary astrocytoma was non-enhancing. Similar non-enhancing astrocytomas have been reported in approximately $9 \%$ of patients with long-segment spinal cord tumors. ${ }^{[7,8]}$ The small enhancing portion in the cord has as differential diagnosis an inflammatory lesion, multiple sclerosis or acute disseminated encephalomyelitis. ${ }^{[8]}$ 


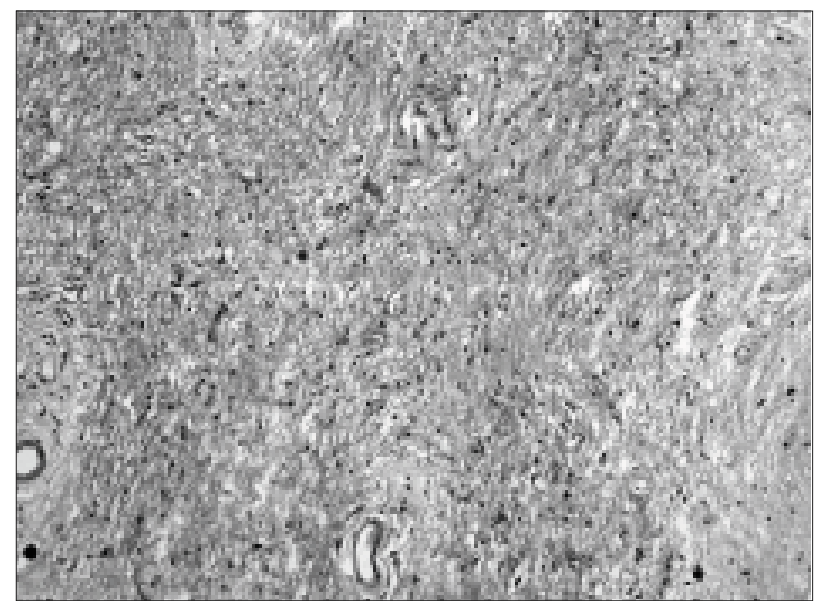

Figure 3: Low-grade astrocytoma having round to oval hyperchromatic nuclei on a fibrillary background and absence of mitotic activity $(\mathrm{H}$ and $E$ $\times 100$, original magnification)

\section{Surgical considerations}

Irreducible AAD is addressed by transoral decompression ${ }^{[2,9,10]}$ and posterior stabilization, usually under the same anesthesia. ${ }^{[9,10]}$ In our patient, however, only posterior decompression of the foramen magnum and assimilated atlas along with C2-5 laminectomy were performed. The intact subarachnoid space anterior to the cord at the C1-2 level despite the T2 cord intensity changes indicated that dynamic neck movements rather than canal compromise was responsible for myelopathy. Thus, occipitocervical stabilization obviated the possibility of further cord trauma by the posteriorly directed odontoid during flexion and extension movements at the neck. The C1-2 lateral mass provided adequate ground for purchase of screws in the presence of suboccipital craniectomy and C2-5 laminectomy. ${ }^{[1]}$ The "two-stage" strategy for radical resection of intramedullary tumors was utilized for addressing the intramedullary tumor. ${ }^{[4]}$ According to this, in the first stage, the midline myelotomy provides the cavity for the poorly-defined tumor to occupy. The tumor filling the myelotomy site may subsequently be safely excised without causing significant postoperative deficits. Thus, tumor decompression was done using suction aspirator but no attempt was made to radically extirpate the tumor considering its strategic location, infiltrative nature and lack of a well-defined plane of cleavage. A duroplasty ensured further space for the cord to expand. This surgical strategy has resulted in

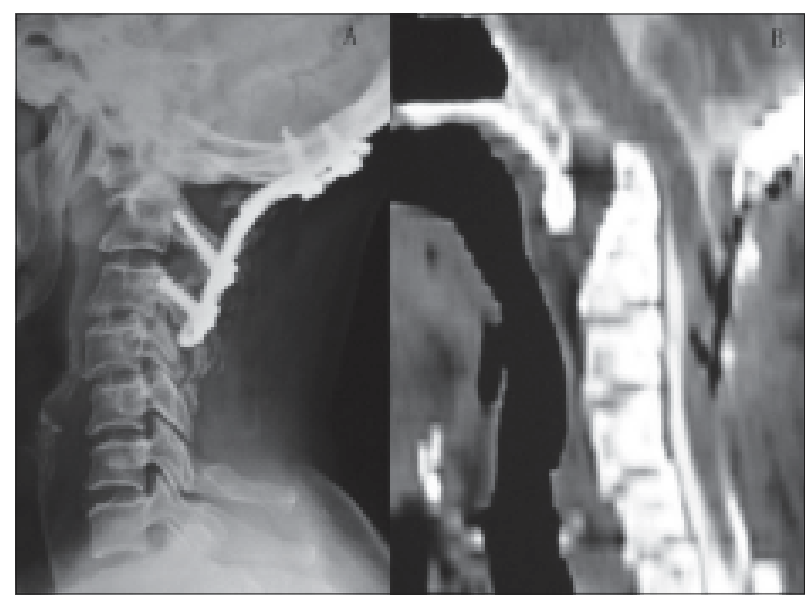

Figure 4: The lateral radiograph $(A)$ and sagittal reconstructed image of intrathecal contrast CT scan of the cervical spine at a follow-up of three months $(B)$ reveals the decompressed posterior margin of the foramen magnum and C2-5 laminectomy. The occipitocervical lateral mass plate and screw construct appears stable

a temporary alleviation of progression of myelopathy. Radiotherapy has been deferred due to the low proliferative potential of the lesion.

\section{References}

1. Behari S, Bhargava V, Nayak S, Kirankumar MV, Banerji D, Chhabra DK, et al. Congenital reducible atlantoaxial dislocation: Classification and surgical considerations. Acta Neurochirur (Wien) 2002;144:1165-77.

2. Jain VK, Behari S. Management of congenital atlanto-axial dislocation: Some lessons learnt. Neurol India 2002;50:386-97.

3. Garrido E, Stein BM. Microsurgical removal of intramedullary spinal cord tumors. Surgical Neurol 1977;7:215-9.

4. Kalangu KK, Couto MT. Radical resection of intramedullary spinal cord tumors without cavitron ultrasonic aspirator or CO2 laser: A "two stage" technique. Surg Neurol 1996;46:310-4.

5. Chhabra R, Bansal S, Radotra BD, Mathuriya SN. Recurrent intramedullary cervical ependymal cyst. Neurol India 2003;51:111-3.

6. Goel A, Muzumdar D, Dange N. Syringomyelia in infants secondary to mobile congenital atlantoaxial dislocation. Pediatr Neurosurg 2007;43:15-8.

7. White JB, Miller GM, Kayton KF, Krauss WE. Nonenhancing tumors of the spinal cord. J Neurosurg (Spine) 2007;7:403-7.

8. Brinar M, Rados M, Habek M, Posner CM. Enlargement of spinal cord: inflammation or neoplasm. Clin Neurol Neurosurg 2006;108:284-9.

9. Crockard HA, Calder I, Ransford AO. One-stage transoral decompression and posterior fixation in rheumatoid atlanto-axial subluxation. J Bone Joint Surg Br 1990;72:682-5.

10. Menezes AH, VanGilder JC, Graf CJ, McDonnell DE. Craniocervical anomalies: A comprehensive surgical approach. J Neurosurg $1980 ; 53: 444-55$

Accepted on 09-05-2008

Source of Support: Nil, Conflict of Interest: None declared. 